\title{
RAF complexities spark caution
}

Several RAF inhibitors are in clinical trials, but three recently published papers indicate that the function of the RAS pathway in a patient's tumour will need to be assessed to avoid potential tumour-promoting effects of these inhibitors.

Although ATP-competitive RAF inhibitors are active against tumours with mutant, constitutively active BRAF(V600E), which generally do not harbour activating RAS mutations, they do not suppress the growth of tumours with mutant RAS or wildtype BRAF. Indeed, RAF inhibitors can activate RAF signalling pathways in some cells. The RAS-RAF pathway is not linear and involves interactions between several kinases. BRAF can bind and activate CRAF in a RASdependent manner, leading to the activation of the downstream kinases MEK and ERK. Most oncogenic mutants of BRAF activate MEK directly; however, some act through CRAF and occasionally BRAF mutants that have no kinase activity are selected for in human tumours.

Richard Marais and colleagues found that drugs that inhibit BRAF result in the binding of BRAF to CRAF and CRAF activation in the presence of activated wild-type or oncogenic RAS. A gatekeeper mutant of BRAF that is much less sensitive

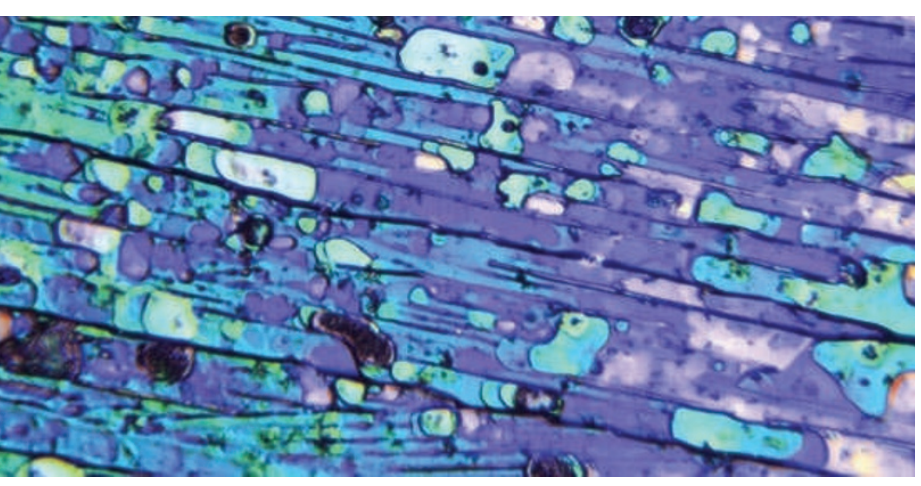

to RAF inhibitors could not dimerize with CRAF, but expression of a CRAF gatekeeper mutant did not prevent binding to BRAF or MEK-ERK activation in the presence of a RAF inhibitor. Use of a kinase-dead mutant of BRAF showed that the kinase activity of BRAF was not needed for BRAF to dimerize with CRAF and activate it. These results indicate that BRAF inhibitors can activate the MEK-ERK pathway because they induce a kinasedead BRAF, which - by a mechanism that involves activated RAS - dimerizes with and induces activation of CRAF. These authors showed that kinase-dead BRAF can cooperate with oncogenic KRAS to induce melanoma in mice, further illustrating the complexities of this pathway.

Whereas BRAF is a key component of the mechanism proposed by Marais and colleagues, Shiva Malek, Georgia Hatzivassiliou and colleagues found that CRAF can be activated by RAF inhibitors in BRAF-null tumour cell lines through the formation of CRAF homodimers. Moreover, activation of MEK was reduced by RAF inhibitors in cells expressing the CRAF gatekeeper mutant that cannot bind these inhibitors. Further investigation showed that RAF inhibitors that transiently bind (fast off-rates) CRAF led to the activation of MEK, whereas a RAF inhibitor with a slow off-rate prevented the activation of MEK by CRAF. Having solved the crystal structure of the CRAF kinase domain bound to an inhibitor, these authors suggest that CRAF activation by ATP-competitive RAF kinase inhibitors probably occurs as a result of inhibitor-mediated modulation of the kinase domain after transient binding. Although Neal Rosen, Kevan Shokat and colleagues also showed that RAF inhibitors result in the activation of CRAF, their model of activation is different. RAF proteins need to bind ATP to be active, and obviously one protein cannot bind both an ATP-competitive inhibitor and ATP at the same time. Instead, these authors showed that binding of the inhibitor to one RAF protein in the dimer results in the transactivation of the other RAF protein. Their results also indicate that RAS activity is required as it promotes the formation of RAF homodimers and heterodimers. This model might also explain why low concentrations of RAF inhibitors lead to the activation of the MEK-ERK pathway, whereas high concentrations result in inhibition.

Despite their differences, all three papers support several conclusions: RAF inhibitors should continue to prove efficacious in tumours with oncogenic BRAF that do not have other changes in the RAS pathway, and so patient screening should be mandatory prior to allocation of the drug; resistance to RAF inhibitors could arise through the activation of RAS or mutations that promote RAF activity; and activation of the MEK-ERK oncogenic signalling pathway by RAF inhibitors might explain the occurrence of squamous cell carcinomas in some patients treated with these drugs in Phase I studies.

Nicola McCarthy, Nature Reviews Cancer

ORIGINAL RESEARCH PAPERS Heidorn, S. J. et al. Kinase-dead BRAF and oncogenic RAS cooperate to drive tumour progression through CRAF. Cell 140, 209-221 (2010) | Poulikakos, P. I., Zhang, C., Bollag, G., Shokat, K. M. \& Rosen, N. RAF inhibitors transactivate RAF dimers and ERK signalling in cells with wild-type BRAF. Nature 23 Feb 2010 (doi:10.1038/nature08902) | Hatzivassiliou, G. et al. RAF inhibitors prime wildtype RAF to activate the MAPK pathway and enhance growth. Nature 3 Feb 2010 (doi:10.10.38/nature08833) 